\title{
Magnetometer-enhanced personal locator for tunnels and GPS-denied outdoor environments
}

\author{
Surat Kwanmuang $^{\mathrm{a}}$, Lauro Ojeda ${ }^{\mathrm{b}}$, Johann Borenstein ${ }^{\mathrm{a}, \mathrm{c}}$ \\ ${ }^{a}$ Dept of Mechanical Engineering, University of Michigan, 2260 Hayward St, Ann Arbor, MI 48109; \\ ${ }^{b}$ Dept of Mechanical Engineering, University of Michigan, 2350 Hayward St, Ann Arbor, MI 48109; \\ cPOC: johannb@umich.edu, ph.: 734-763-1560
}

\begin{abstract}
This paper describes recent advances with our earlier developed Personal Dead-reckoning (PDR) system for GPS-denied environments. The PDR system uses a foot-mounted Inertial Measurement Unit (IMU) that also houses a three axismagnetometer. In earlier work we developed methods for correcting the drift errors in the accelerometers, thereby allowing very accurate measurements of distance traveled. In addition, we developed a powerful heuristic method for correcting heading errors caused by gyro drift. The heuristics exploit the rectilinear features found in almost all manmade structures and therefore limit this technology to indoor use only.

Most recently we integrated a three-axis magnetometer with the IMU, using a Kalman Filter. While it is well known that the ubiquitous magnetic disturbances found in most modern buildings render magnetometers almost completely useless indoors, these sensors are nonetheless very effective in pristine outdoor environments as well as in some tunnels and caves.

The present paper describes the integrated magnetometer/IMU system and presents detailed experimental results. Specifically, the paper reports results of an objective test conducted by Firefighters of California's CAL-FIRE. In this particular test, two firefighters in full operational gear and one civilian hiked up a two-mile long mountain trail over rocky, sometimes steeply inclined terrain, each wearing one of our magnetometer-enhanced PDR systems but not using any GPS. During the hour-long hike the average position error was about 20 meters and the maximum error was less than 45 meters, which is about $1.4 \%$ of distance traveled for all three PDR systems.
\end{abstract}

Keywords: Personal locator, tunnels, GPS-denied, dead-reckoning, gyro, drift, position

\section{INTRODUCTION}

This paper describes a system for estimating the position of walking persons in outdoor or underground environments, in which GPS is not available and magnetic disturbances are few. The system comprises our earlier-developed Personal Dead-reckoning (PDR) system and a newly developed magnetometer-based software module. The former is fully discussed in our earlier publications [1],[2],[3], but for completeness, we provide a brief description in Section 1.1. The PDR system uses a foot-mounted Inertial Measurement Unit (IMU), which includes a three-axis gyroscope, a three-axis accelerometer, and a three-axis magnetometer. In earlier work, we used only gyroscopes and accelerometers for position estimates and developed a method for correcting accelerometer drift that exploited the fact that an instrumented human foot briefly experiences zero velocities during every footfall. In addition and more recently, we developed a heuristic method for correcting heading errors caused by drift and other physical phenomena in the gyroscopes. The heuristic method exploits the rectilinear features in man-made structures. However, this method is useful only inside buildings that have rectilinear features.

For most outdoor environments, GPS is the most accurate and cheapest solution [4] for all but the shortest of walks. However, for many military applications it is desirable not to rely on GPS, and in some other applications GPS may be unreliable due to occlusion (e.g., under dense tree canopies or in canyons). In tunnels and caves GPS is altogether not 
available. Local GPS-like beacons (also called pseudolites) can provide centimeter-accuracy over areas of several square miles, but the pseudolites have to be preinstalled before a mission and even then don't penetrate well into structures [5].

Unlike gyros, which estimate relative changes in heading, a sensor modality with the ability to estimate absolute heading is the magnetometer. The earth's magnetic properties have been known and used in compasses for navigation for hundreds of years. Modern electronic magnetometers are used extensively in aviation and watercrafts for measuring heading with respect to the Earth's magnetic north. However, magnetometers are affected by magnetic disturbances (as will be discussed in more detail) and are generally not reliable inside modern structures. Nonetheless, for pristine, natural outdoor environments magnetometers are very suitable for bounding the otherwise unlimited growth of heading errors derived from gyros.

Much research on the use of magnetometers for personal positioning applications has been conducted in recent years. Some approaches use magnetometers exclusively for heading estimation [6], while others integrate it tightly with an IMU [7],[8].

One commercially available personal locator system based on this principle is the Dead Reckoning Module DRM-4000 made by Honeywell [9]. The DRM-4000 uses accelerometers to identify steps, and linear displacement is estimated by what Honeywell describes as: "motion classification algorithms that analyze walking motion and compensate for unique user kinematics." Using these algorithms, Honeywell claims an accuracy of $2 \%$ of the traveled distance. However, anecdotal observations from users of the Honeywell system seem to suggest that this performance is achieved only under the most ideal conditions and rarely in practice.

\subsection{The Personal dead-reckoning system}

The magnetometer-supported navigation system discussed in this paper is based on our existing Personal Deadreckoning (PDR) system, enhanced by the newly developed magnetometer component that is the main focus of this paper. For completeness, we provide here a summary of the functionality of the PDR system.

The PDR system uses an IMU strapped to the side or embedded in the heel of the user's boot, as shown in Figure 1. The side-mounted IMU can be transferred easily among different users while the in-heel version better protects the IMU from damage and cannot be dislocated easily. The PDR system's computations are performed on a PC-104 computer that is located inside a belt pack, together with batteries and support electronics. The MEMS-based IMU used in the PDR system is the nano-IMU ("nIMU" in short), made by Memsense. Some key specifications for the nIMU are listed in Table 1.

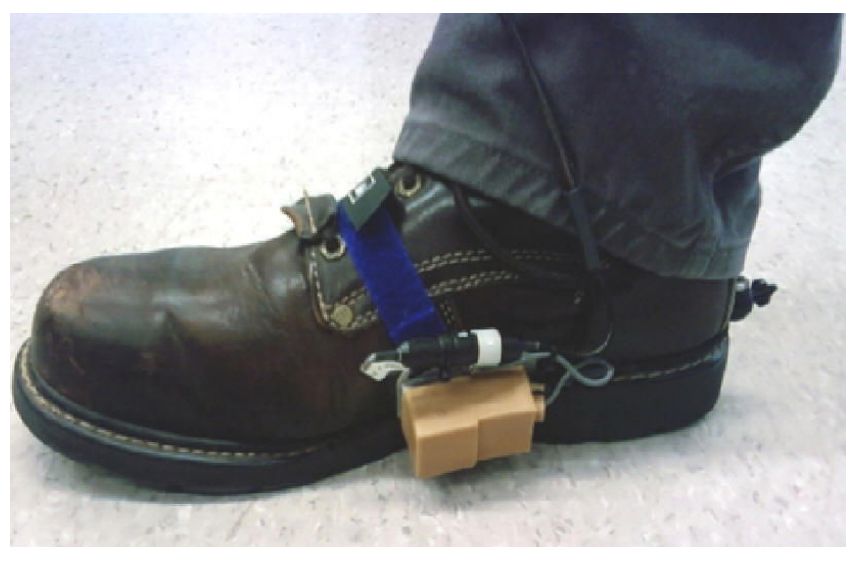

(a)

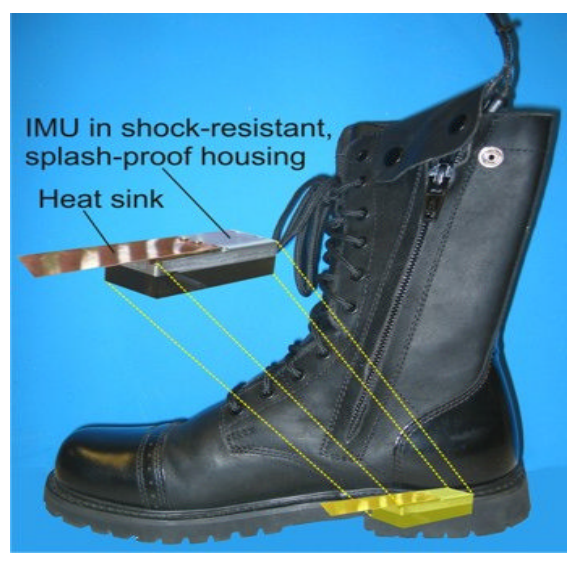

(b)

Figure 1. The foot-mounted IMU of the PDR system has two mounting options. (a) Side-mounted IMU (the IMU itself is covered by the beige-colored thermal insulation). (b) In-heel IMU with temperature controlled, shock resistant housing 
Table 1. Key Specifications of the Memsense nIMU

\begin{tabular}{|l|l|}
\hline \multicolumn{2}{|l|}{ Memsense nIMU } \\
\hline Size (mm) & $45 \times 23 \times 13$ \\
\hline Weight (gram) & 15 \\
\hline Bandwidth (Hz) & 75 \\
\hline Gyroscope & $\pm 1,200$ \\
\hline Range (deg/sec) & 4.2 \\
\hline Angle Random Walk (deg/sqrt-hr) & 80 \\
\hline Bias Drift (deg/hr) & \pm 10 \\
\hline Accelerometer & \\
\hline Range (g) & \\
\hline
\end{tabular}

An IMU-based position estimation system combines two functions: the estimate of distance traveled and the estimate of heading. In the PDR system, the accuracy of both components is predominantly affected by bias drift ("drift," in short). This is especially true if a relatively low-performance MEMS-based IMU is used. Drift rates for both accelerometers and gyroscopes in a MEMS-based IMU are several orders of magnitude higher than what is found in high-grade aviation IMUs. Of course, the cost of high-grade IMUs is also several orders of magnitude higher than that of the nIMU in the PDR system. However, the reason for using a low-grade IMU in the PDR system is not just cost. Rather, in order to embed the IMU in the heel of a regular firefighter or military-style boot, the device must be very small. Because of this size-limitation, the only suitable IMU technology is that of MEMS. Another limiting factor is the fact that the peak accelerations and rates of turn of the foot, even at normal walking speed, are significantly higher than those found on the torso of a person. This limits the choice of suitable IMUs to less than a handful of models that offer the large dynamic range required by that particular IMU location.

A foot-mounted IMU makes wiring more difficult, requires greater dynamic ranges of the IMU, and may add difficulties in heading estimation. However, there is a compelling reason for choosing this mounting location after all: it is the only way to estimate drift of the accelerometers and subtract this drift from subsequent readings almost as frequently as once every second. This method of resetting drift is called Zero Velocity Updates (ZUPT) and it is possible with a footmounted IMU because the instrumented foot has indeed zero velocity during a brief moment while the foot is fully in contact with the floor. We call the moment of zero foot velocities "footfall." Human walking has the uniquely favorable property of allowing ZUPT to be performed naturally, without having to halt motion, and as frequently as once every other step at a rate of about $1 \mathrm{~Hz}$. Because ZUPT can be applied so frequently, errors due to accelerometer drift can be eliminated almost entirely. As a result, the estimation of distance traveled has consistently errors of only $1 \%$ of distance traveled. Moreover, since the PDR system actually estimates the distance and direction of the foot motion, there is no need to calibrate the system for each user. Also, the PDR system works equally well when users walk sideways, backwards, or in uncommon gaits. A more complete discussion of the function of the PDR system is provided in [1],[2].

\section{THE MAGNETOMETER ENHANCED PERSONAL LOCATOR SYSTEM}

One remaining problem is the substantial drift of the gyros, which result in heading errors of $80 \mathrm{deg} / \mathrm{s}$ and more. In earlier work we developed one method that is very effective for eliminating errors due to gyro drift, called Heuristic Drift Elimination (HDE) [3]. However, this method only works inside buildings with rectilinear walls and corridors.

Since HDE cannot be applied to outdoor walking, another sensor modality is needed to counteract the effects of gyro drift. The magnetometer is a good choice because - although not very useful inside modern buildings - it works very well in pristine outdoor environments.

For the purpose of discussing the main subject of this paper, integration of a magnetometer component, we consider the PDR system as a black box. This black box outputs position and heading of the walker in real-time and at footfall 
intervals. Also available at every instance of footfall are the ZUPT-corrected accelerometer readings. Since the instrumented foot is stationary during footfalls, we can exploit the fact that the only acceleration affecting the accelerometers at this instance is that of gravity. Consequently, we can determine roll and pitch, collectively called "tilt" of the IMU (which also houses the magnetometer) with good accuracy at the moment of footfall. Using tilt estimates based on accelerometers allows us to estimate and correct tilt errors.

The remaining problem is estimating heading errors. In order to simplify the discussion of this topic we introduce the term "virtual gyro." The output of the virtual z-axis gyro is rate of turn around the world z-axis. If we were able to estimate the virtual drift of the virtual z-axis gyro (which is, of course, the result of real drift in the three real gyros), then we could correct all real gyro drift errors since we already have reasonably good estimates for the drift of $\mathrm{x}$ and $\mathrm{y}$-axis gyros from the accelerometers. Thus, in the remainder of this paper, we can limit our discussion to the problem of estimating virtual Z-axis gyro drift. One advantage of treating the PDR system as a black box, instead of trying to integrate the magnetometer with the full set of mechanization equations, is that the proposed magnetometer functionality is modular and easy to port to other applications. Within the PDR system, the modular approach makes it easy to switch the magnetometer module "on" or "off," for example, when switching to the HDE module when the user enters a building.

\subsection{The magnetometers}

A three-axis magnetometer, such as the one built into the Memsense nIMU used in our PDR system, can determine the 3-dimensional direction of the magnetic field around the sensor. Absolute heading can be measured by decomposing the 3-dimensional magnetic field vector into three components, $H_{x}, H_{y}, H_{z}$ that are aligned with the navigation frame of the IMU

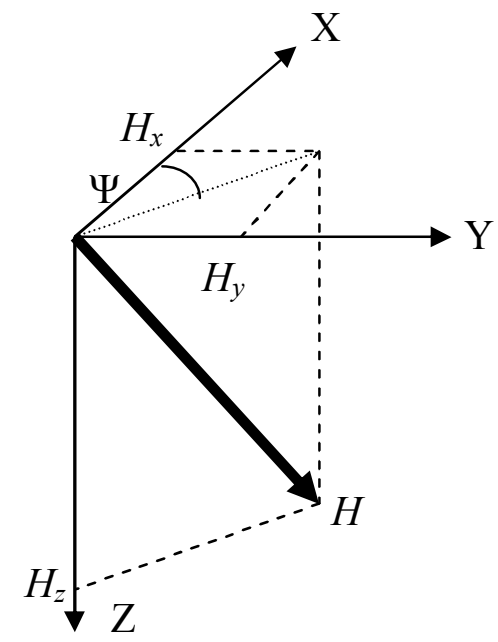

Figure 2. The graphical visualization of the Earth's magnetic field measurement $H$ from magnetometers. $\Psi$ is the heading with respect to the magnetic north.

$$
\Psi=-\operatorname{atan} 2\left(H_{y}, H_{x}\right)
$$

Where $H$ is the magnetic field in the IMU's navigation frame (the $\mathrm{X}$-axis coincides with the magnetic north and the Y-axis points east). However, since the measurement is taken in the IMU's body frame (where the X-axis points forward and the Y-axis points to the right of the sensor) the IMU's pose has to be known or estimated so that the sensor reading in the body frame can be transformed into the navigation frame. Thus, any misalignment between the magnetometer component and the other sensors (notably the accelerometers, which estimate tilt at footfalls) will contribute to the heading calculation error. 


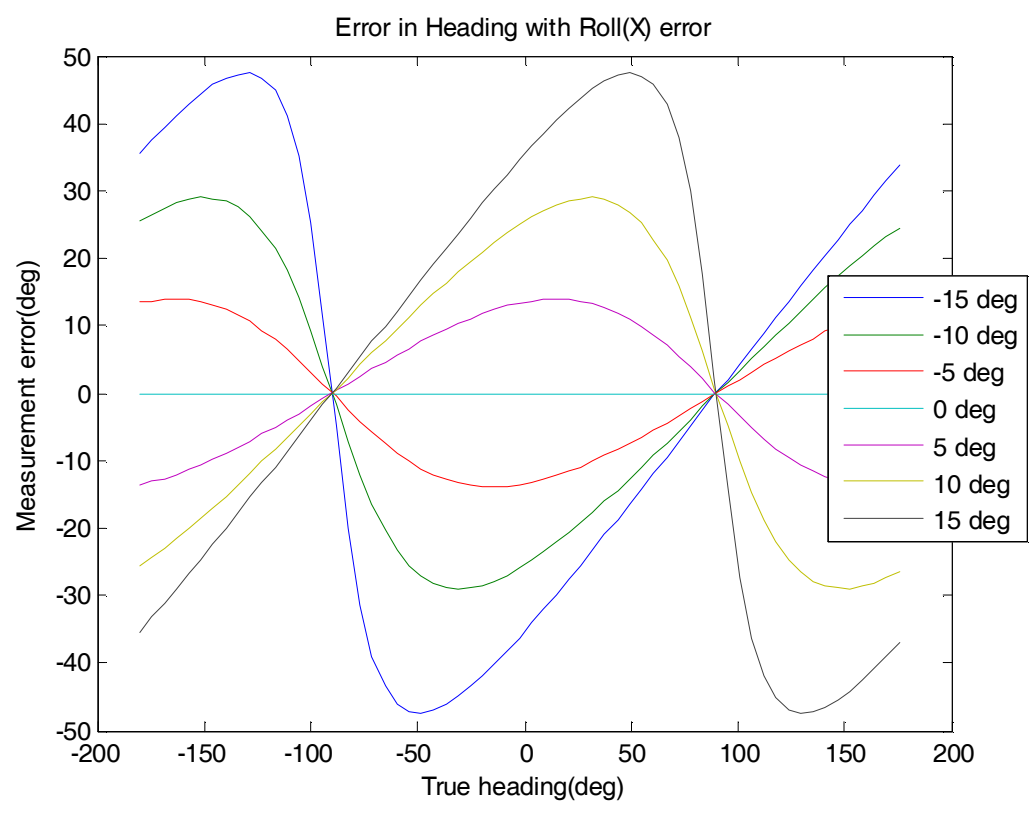

Figure 3. Heading measurement error due to misalignment in various roll angles

Additional errors result from local magnetic disturbances, such as from electro-magnetic fields around power lines, electronics, and steel structures. These errors can be divided into hard and soft iron errors. We only list these error sources below; a more exhaustive discussion is provided in [10]. For all magnetometer applications, accuracy can be improved significantly by performing a calibration procedure prior to each trip as will also be discussed, below.

\subsection{Typical errors with magnetometers}

\section{- Hard Iron effect}

The hard iron effect is caused by nearby ferromagnetic materials that produce a constant additive magnetic field. This effect results in an offset in the sensor measurement.

\section{- Soft Iron effect}

The soft iron effect is caused by some materials that change the magnetic field depending on the orientation of the sensor. This error distorts measurements from the magnetometer as a function of sensor heading.

\section{- Misalignment effect}

To some degree, the different sensor modalities inside the IMU are misaligned relative to each other. This will result in crossover measurements in all sensor axes.

\section{CALIBRATION PROCEDURE}

The calibration procedure for the magnetometer is arranged into two steps. The first step is to eliminate sensor misalignment, while the second step aims at correcting hard and soft iron errors of the sensor. 


\subsection{Calibration Steps}

\subsection{Step 1 - Misalignment correction}

When rotating the magnetometer horizontally, misalignment causes the magnetometer's Z-axis to be different from the world Z-axis. This causes the measured field vectors to be located on an inclined plane that corresponds to the misalignment angle. To remove this misalignment, we fit a plane to all measurements using a linear least square approach. Then, we rotate the plane so that the normal of the plane is parallel to the world Z-axis.

Equation of a plane:

$$
a H_{x}+b H_{y}+c H_{z}+d=0
$$

The normal to this plane is defined as

$$
\vec{n}=\left[\frac{a}{\sqrt{a^{2}+b^{2}+c^{2}}}, \frac{b}{\sqrt{a^{2}+b^{2}+c^{2}}}, \frac{c}{\sqrt{a^{2}+b^{2}+c^{2}}}\right]^{T}
$$

In order to rotate the normal vector to make it vertical, a rotation axis and rotation angle can be found by computing

$$
\begin{gathered}
\vec{r}=\vec{n} \times[0,0,1]^{T}=\left[n_{y},-n_{x}, 0\right]^{T} \\
\theta=\arccos \left(\frac{\vec{n}}{\|\vec{n}\|}\right)
\end{gathered}
$$

By rotating all subsequence measurements through this angle along the rotation axis, we can correct for the misalignment of the magnetometer.

\subsection{Step 2 - Soft iron and hard iron calibration}

The second step of the calibration process exploits the fact that in a disturbance-free environment and after performing Step 1, a full rotation around the Z-axis should yield magnetic field vectors of identical magnitude. One can picture this condition as the locus of the tip of all measured field vectors. Since the magnitude of the horizontal magnetic field, $H_{x y}$, is constant, this locus should ideally be a circle.

$$
H_{x}{ }^{2}+H_{y}{ }^{2}=\left(\vec{H}_{x y} \cos (\Psi)\right)^{2}+\left(\vec{H}_{x y} \sin (\Psi)\right)^{2}=\left\|\vec{H}_{x y}\right\|^{2}
$$

However, in practice the hard and soft iron errors will distort that circle. Specifically, the hard iron disturbances move the center of the circle, and the soft iron effects distort the shape of the circle to that of an ellipse.

An overly optimistic assumption is that there is no crossover effect between the axes of the magnetometer [10]. However, in practice, there is crossover interaction, and as a result the ellipse becomes a tilt ellipse.

We can find the center of the tilted ellipse that has been displaced by the hard iron effects, by using a linear least square approach:

The general equation for tilted ellipse with center at $\left[x_{c}, y_{c}\right]$ has this form

$$
\begin{gathered}
a\left(x-x_{c}\right)^{2}+b\left(y-y_{c}\right)^{2}+c\left(x-x_{c}\right)\left(y-y_{c}\right)=1 \\
a x^{2}-2 a x x_{c}+a x_{c}{ }^{2}+b y^{2}-2 b y y_{c}+b y_{c}{ }^{2}+c x y+c x_{c} y_{c}-c x x_{c}-c y y_{c}=1
\end{gathered}
$$

By assuming $\left[x_{c}, y_{c}\right]$ are small, we can remove quadratic terms 


$$
a x^{2}+b y^{2}+c x y-(2 a+c) x_{c} x-(2 b+c) y_{c} y=1
$$

Defining

$$
d=-(2 a+c) x_{c} \quad \text { and } \quad e=-(2 b+c) y_{c}
$$

we can now rewrite the linear equation of the ellipse

$$
a x^{2}+b y^{2}+c x y+d x+e y=1
$$

By solving Eq. (11) using least squares, the displacement of the center of the ellipse can be found

$$
x_{c}=\frac{d}{-(2 a+c)} \quad \text { and } \quad y_{c}=\frac{e}{-(2 b+c)}
$$

The equation for the same ellipse but with its center at the origin has this form

$$
a x^{2}+b y^{2}+c x y=1
$$

Eq. (13) can be written in matrix form

$$
\left[\begin{array}{l}
x \\
y
\end{array}\right]^{T}\left[\begin{array}{cc}
a & c / 2 \\
c / 2 & b
\end{array}\right]\left[\begin{array}{l}
x \\
y
\end{array}\right]=\left[\begin{array}{l}
x \\
y
\end{array}\right]^{T} M\left[\begin{array}{l}
x \\
y
\end{array}\right]=1
$$

In order to estimate the soft iron effect, we can decompose the matrix $M$ using Singular Value Decomposition $M=U \cdot \Sigma \cdot V^{*}$. Then, the semi-major and semi-minor axes are the columns of the nearest orthonormal matrix of $M$, which is $A=U \cdot V^{*}$. The length of the ellipse is given by the diagonal components of $\Sigma$.

By combining the above estimations, we can correct subsequent measurements

$$
\left[\begin{array}{l}
H_{x}{ }^{\text {corrected }} \\
H_{y}{ }^{\text {corrected }}
\end{array}\right]=A\left[\begin{array}{cc}
\frac{1}{\Sigma_{11}} & 0 \\
0 & \frac{1}{\Sigma_{22}}
\end{array}\right] A^{T}\left[\begin{array}{l}
H_{x}-x_{c} \\
H_{y}-y_{c}
\end{array}\right]
$$

After performing these corrections, one can estimate heading from Equation (1).

\subsection{Robust estimation}

In practice, apart from the local magnetic field, there are magnetic disturbances and noise that are also collected during calibration. Since the least square estimation of Eqs. (2) and (11) treats all samples equally, noise will add bias to the estimation. To cope with this problem, the maximum likelihood estimation can be used instead. In our implementation, we use the Huber estimation [11] with good success. 


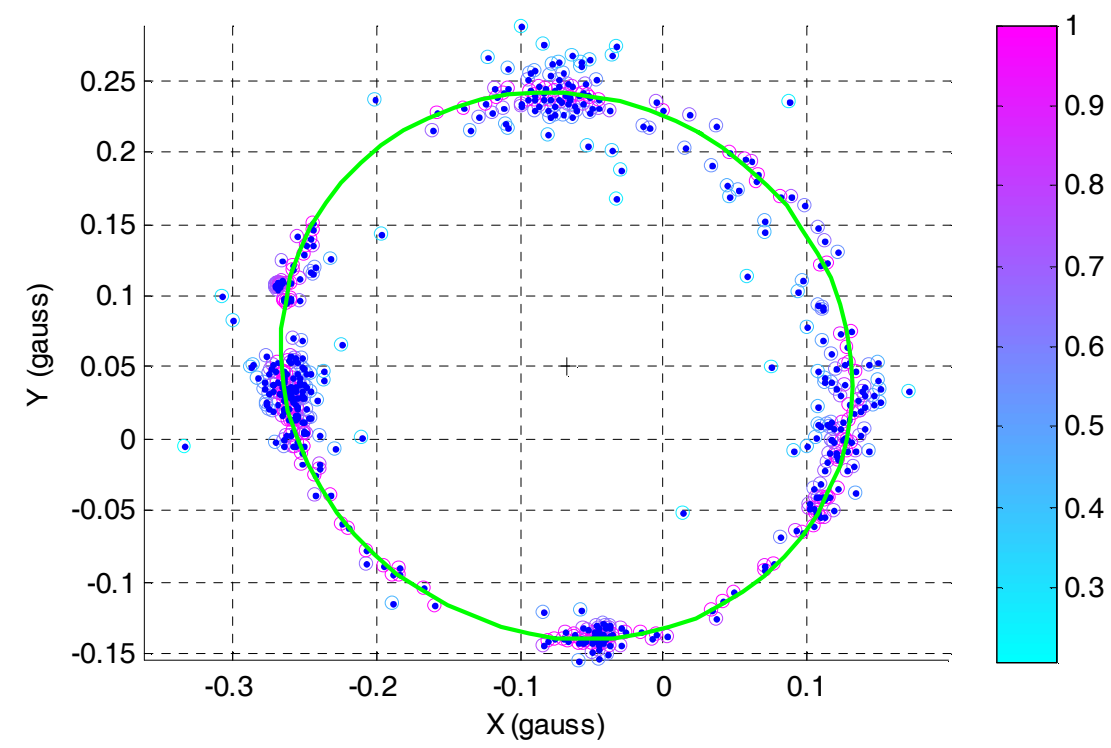

Figure 4. Result of soft/hard iron calibration with robust estimation. The blue dots are magnetic field measurements $H_{x}, H_{y}$ from the magnetometers. The color of the small circles around each measurement represent the weight of each point from the Huber estimation. The black crosshair near the center shows the estimated displacement of the center due to the hard iron effect and the green ellipse shows the distortion of the ideal circle due to the soft iron effect.

\subsection{Calibration procedure}

For best results, a calibration of the magnetometer has to be performed once in the beginning of every walk. The calibration procedure requires the user to walk around in a circle for at least one full circle. The walk does not have to follow a circular contour with any accuracy; the only requirement is that the magnetometer experiences a full 360-degree rotation around the vertical axis. As a concession to practicality, we implemented the calibration process so that the system performs it as a background task. In practice, the user can start a walk anywhere, without performing the calibration circle immediately at the beginning of the walk. The PDR system will track the user immediately, albeit with the magnetometer calibrated with the calibration data from the previous walk. Then, when the user has an opportunity and a suitable magnetically undisturbed location, the user can perform the calibration circle and continue with the mission. The system automatically notices when a full circle has been completed, performs the mathematical operations needed for the calibration, and then uses the fresh calibration data from here on out.

\section{REAL-TIME ALGORITHM}

\subsection{Magnetic disturbance detection}

During operation, especially inside modern buildings and in urban environments, a magnetometer is subject to many external disturbances. For example, since our magnetometer is mounted in the heel of the user's footwear, large metal objects such as manhole covers or underground pipes can create large disturbances. To minimize the effect of external magnetic disturbances, our system attempts to detect the presence of these temporary disturbances. Once detected, the affected magnetometer readings can be discarded and replaced by IMU-derived heading estimates.

To detect magnetic disturbances, we monitor two criteria:

\section{Magnetic field strength}

If the measured field strength deviates by more than a predefined difference from the theoretical field strength based on the International Geomagnetic Reference Field (IGRF11) model [12], the measurement is flagged as a magnetic disturbance. However, in order to use the IGRF11 model, the local latitude and longitude have to be known. In practice, 
we substitute for the theoretical IGRF11 model the average of measured field strengths during start up. This is reasonable since we work under the assumption that the system is initialized in an area that has none or only small magnetic disturbances. We found empirically that this average provides sufficient accuracy and it also eliminates the need for users to enter the local coordinates into the system.

\section{Rate of turn}

In order to apply this criterion, we compare the rate of turn derived from changes in the magnetometer heading to the rate of turn computed from the gyros in the PDR system. If they disagree, the magnetometer measurement is flagged as a disturbance.

For both criteria, once a disturbance is flagged the flag stays in effect for several subsequent magnetometer measurements. We chose this conservative approach because it is preferable to lose a few measurements rather than introduce erroneous magnetometer data into the system.

\subsection{Sensor fusion}

The position output from the IMU of the PDR system is not very noisy. However, heading errors grow without bound because of drift in the gyros. In contrast, heading derived from the magnetometer has no drift but has substantial noise. To fuse data from these two disparate sensor modalities, we designed a Kalman filter [13]. We formulate the state space equations assuming that the PDR heading is affected by random walk bias drift.

The states of the system that we want to estimate are heading and bias:

$$
\begin{gathered}
\hat{x}_{k+1}=A \hat{x}_{k}+B \vec{u}_{k} \\
\hat{x}_{k+1}=\left[\begin{array}{c}
\Psi \\
\text { bias }
\end{array}\right]_{k+1}=\left[\begin{array}{cc}
1 & -\Delta t \\
0 & 1
\end{array}\right]\left[\begin{array}{c}
\Psi \\
\text { bias }
\end{array}\right]_{k}+\left[\begin{array}{l}
1 \\
0
\end{array}\right] \Delta \Psi_{P D R}
\end{gathered}
$$

The covariance error is predicted using:

$$
P_{k+1}=A P_{k} A^{T}+Q
$$

The measurement equation has the form

$$
z=\Psi_{\text {mag }}=H \hat{x}_{k}=\left[\begin{array}{ll}
1 & 0
\end{array}\right]\left[\begin{array}{c}
\Psi \\
\text { bias }
\end{array}\right]_{k}
$$

When a measurement is available from the magnetometer, the system is updated using:

$$
\begin{aligned}
& K_{k}=P_{k} H^{T}\left(H P_{k} H^{T}+R\right)^{-1} \\
& \hat{x}_{k}=\hat{x}_{k}+K_{k}\left(z_{k}-H \hat{x}_{k}\right) \\
& P_{k}=\left(I-K_{k} H\right) P_{k}
\end{aligned}
$$

The correction part of the filter is applied only when the disturbance flag is down. If the flag is up, the system uses only prediction data. 


\section{EXPERIMENTAL RESULTS}

The PDR system and its integrated magnetometer were tested by firefighters from the California Department of Forestry \& Fire Protection (CAL FIRE) [14] under the patronage of the Center for Commercialization of Advanced Technology (CCAT) [15]. The test area was Stone Wall Peak, a mountainous hiking trail about $3.2 \mathrm{~km}$ (2.0 miles) long and located near San Diego, California, USA.

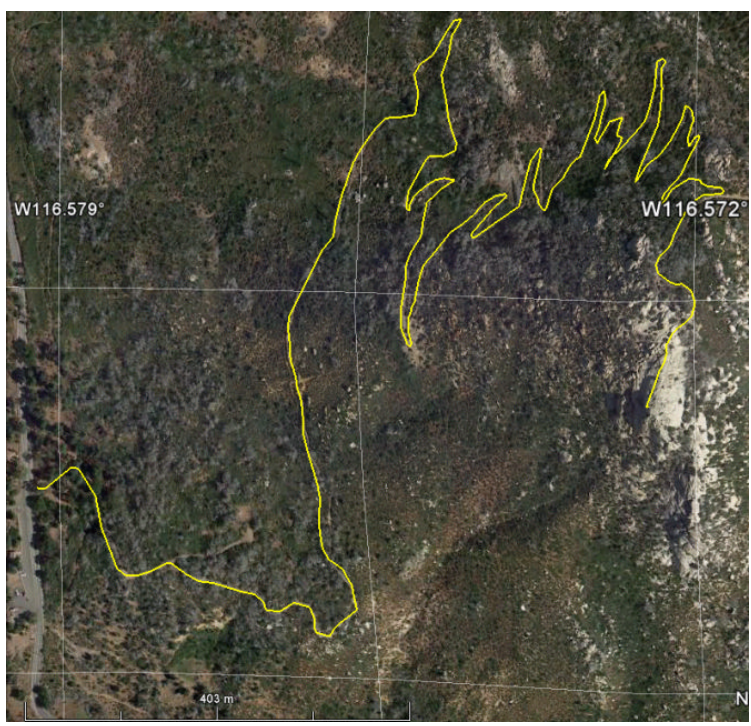

Figure 5. Nominal path as recorded with a handheld GPS receiver in an earlier walk.

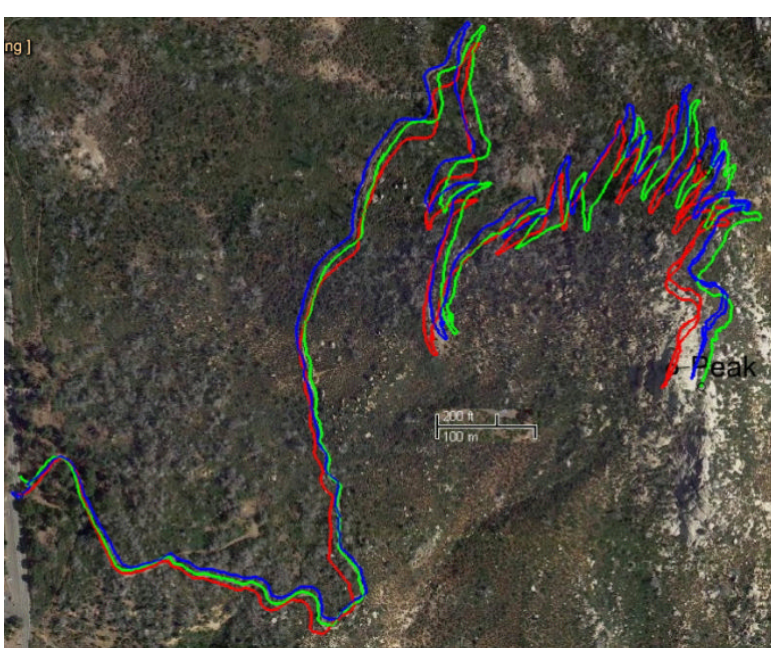

Figure 6. Trajectories as recorded by all three PDR units using IMU and magnetometer only. The actual trail is only faintly visible and is mostly obscured by the three trajectories.

The nominal path, recorded by CAL FIRE firefighters a few weeks ahead of the test (with a handheld GPS unit), is shown as the yellow line overlaid over the Google Earth satellite image of Figure 5. Two firefighters were wearing PDR systems with boots that were instrumented with IMUs. Their trajectories were displayed on an Operator Control Unit $(\mathrm{OCU})$ in red and green colors, and, for simplicity, we refer to them as the red and green PDR systems or the red and green walkers, respectively. One additional experimenter walked the whole trail with a third PDR unit that used a strapon IMU that produced blue trajectories.

Visual examination of the resulting trajectories, shown in Figure 6, suggest that none of the position errors of any one of the three tested PDR systems exceeded $\sim 45$ meters $(=1.4 \%$ of distance traveled), and most of the time position errors were much smaller, less than 20 meters. These are rather remarkable results, given the length, ruggedness, and partially steep inclination of the terrain, as well as the duration of the hike of more than one hour in each direction. Indeed, for the most part, the performance of all three PDR units came closed to that of consumer grade GPS units.

An animated trajectory plot is available at:

http://www.engin.umich.edu/research/mrl/video/StoneWall1280x720.wmv 


\section{CONCLUSIONS}

This paper introduced the magnetometer module of our personal locator system. We found that in a pristine environment and after proper initial calibration, the magnetometer is capable of bounding heading errors from the IMU very effectively. In combination with our existing PDR system, which almost completely eliminates accelerometer drift by mounting the IMU in in the heel of the user's boot, position errors are effectively limited to less than $1.5 \%$ of distance traveled. Trajectories recorded simultaneously by the three tested systems on three users show remarkable agreement with each other and demonstrate the robustness of the systems. We conclude that with the help of the magnetometer module, our PDR system is capable of tracking the position of walkers on somewhat rugged terrain for periods of tens of minutes and more. To be clear, GPS is still the prefered sensor modality for outdoor hikes. However, in applications where GPS satellites may be temporarily occluded or the possibility exists for intentional GPS jamming, the magnetometer-enhanced PDR system can fill in with rather accurate position data during times of GPS outages.

\section{ACKNOWLEDGEMENTS}

This work was supported by the Center for Commercialization of Advanced Technology (CCAT) under Subward Agreement\# 55961A 7838.

\section{REFERENCES}

[1] Ojeda, L., Borenstein, J., 2007, "Non-GPS Navigation for Security Personnel and Emergency Responders.” Journal of Navigation. Vol. 60 No. 3, September 2007, pp. 391-407.

[2] Ojeda, L. and Borenstein, J., 2007, "Personal Dead-reckoning System for GPS-denied Environments." IEEE International Workshop on Safety, Security, and Rescue Robotics (SSRR2007) in Rome, Italy, September 27-29, 2007.

[3] Borenstein, J, Ojeda, L., and Kwanmuang, S, 2009, "Heuristic Reduction of Gyro Drift in a Personal Deadreckoning System." Journal of Navigation, Vol 62, No 1, January 2009, pp. 41-58.

[4] Hofmann-Wellenhof, B., H. Lichtenegger, et al. 1994. "GPS: Theory and practice", Springer.

[5] Cobb H.S., 1997, "GPS pseudolites: Theory, design, and applications", PhD Dissertation, Stanford University.

[6] Cho, S.Y., Lee, K.W., Park, C.G., and Lee, J.G., 2003. "A Personal Navigation System Using Low-Cost MEMS/GPS/Fluxgate." Proceedings of the 59th Institute of Navigation (ION) Annual Meeting, Albuquerque, NM.

[7] Aparicio, C., S. Reader, et al., 2004, "Implementation of a Quaternion-Based Kalman Filter for Human Body Motion Tracking Using MARG Sensors." master's Thesis, Naval Postgraduate School, Monterey, CA.

[8] Yun, X. and E. Bachmann, 2006, "Design, implementation, and experimental results of a quaternion-based kalman filter for human body motion tracking." IEEE Transactions on Robotics 22(6): 1216-1227.

[9] Honeywell DRM4000 , http://www51.honeywell.com/aero/common/documents/myaerospacecatalogdocuments/Missiles-Munitions/DRM4000.pdf, Last accessed: 03/11

[10]D. Gebre-Egziabher, G. H. Elkaim, J. D. Powell, and B. W. Parkinson., 2001, "A non-linear, two-step estimation algorithm for calibrating solid-state strapdown magnetometers." In 8th International St. Petersburg Conference on Navigation Systems, St. Petersburg, Russia. IEEE/AIAA, 2001.

[11] Huber, P. J., E. Ronchetti, et al., 1981, "Robust statistics", Wiley Online Library.

[12]Finlay, C., S. Maus, et al., 2010, "International geomagnetic reference field: the eleventh generation." Geophysical Journal International.

[13] Welch, G. and G. Bishop, 1995, "An introduction to the Kalman filter." University of North Carolina at Chapel Hill, Chapel Hill, NC 7(1).

[14] CAL FIRE - California Department of Forestry \& Fire Protection, http://www.fire.ca.gov/

[15]CCAT - Center for Commercialization of Advanced Technology, http://www.ccatsandiego.org/ 